\title{
Pré-Diagnóstico da Doença Fúngica Antracnose no Cultivo do Capsicum Annuum Através da Lógica Fuzzy
}

\author{
Roney Nogueira de Sousa ${ }^{1}$, Rhyan Ximenes de Brito ${ }^{1}$, \\ Janaide Nogueira de Sousa Ximenes ${ }^{2}$ \\ ${ }^{1}$ Instituto Federal de Educação, Ciência e Tecnologia do Ceará (IFCE) \\ CE-187, s/n - Estádio, - CEP 62320-000 - Tianguá - CE - Brasil \\ ${ }^{2}$ Faculdade IEducare (FIED) - Rua Conselheiro João Lourenço, \\ 406 - CEP 62320-000 - Tianguá - CE - Brasil \\ \{nogueiraroney453,rxbrito, nogueirajanaide\}@gmail.com
}

\begin{abstract}
The use of Computational Intelligence techniques has been highlighted in solving the most diverse types of problems. Based on this perspective, a study was carried out through the implementation of a Fuzzy system aiming to use it as a diagnostic tool for anthracnose disease in a pepper garden. The methodology was based on the collection of samples of plants with characteristics of anthracnose disease used as input variables in Fuzzy. Three diagnostic tests were carried out with collected data, showing to be quite promising compared to the expected. The study showed the importance of using Fuzzy logic to aid in the detection of diseases such as anthracnose that attacks vegetables such as peppers.
\end{abstract}

Resumo. A utilização de técnicas de Inteligência Computacional tem-se destacado na resolução dos mais diversos tipos de problemáticas. Com base nessa perspectiva realizou-se um estudo através da implementação de um sistema Fuzzy objetivando usá-lo como ferramenta de diagnóstico da doença antracnose em uma horta de pimentões. A metodologia baseou-se na coleta de amostras de plantas com características da doença antracnose usadas como variáveis de entradas no Fuzzy. Realizou-se 3 testes de diagnósticos com dados coletados, mostrando-se bastante promissores face ao esperado. O estudo mostrou a importância da utilização da lógica Fuzzy no auxílio da detecção de doenças como a antracnose que ataca hortaliças como os pimentões.

\section{Introdução}

É sabido que o pimentão pertence a família das solanáceas sendo cultivado em clima tropical e está entre as dez hortaliças mais importantes do mercado brasileiro [da Silva Albuquerque et al. 2011], porém produtores sofrem com perdas devido a algumas doenças, entre elas destacando-se a antracnose que atinge todos os estágios de desenvolvimento da planta, desde mudas no viveiro até os frutos após a colheita [de Azevedo et al. 2006].

A antracnose pode ser considerada uma doença comum, de ocorrência generalizada no Brasil, especialmente quando o período de cultivo coincide com chuvas e a incidência de clima quente e úmido [de Azevedo et al. 2006]. 
O estudo em questão visa implementar e analisar os dados obtidos através de um sistema baseado em lógica Fuzzy visando usá-lo como auxílio no diagnóstico da doença antracnose. A metodologia baseou-se no aprofundamento bibliográfico assim como na coleta de amostras de plantas com características da doenca antracnose que foram usadas como variáveis de entrada observando os sintoma apresentados no caule, folhas e frutos de plantas infectadas. Os resultados foram analisados de acordo com os resultados da saída gerada pelo sistema, com grau de verdade variando de baixíssimo a altíssimo.

A motivação para o presente estudo está relacionada a necessidade de aprimoramento de técnicas para a diagnose efetiva e precoce da doença antracnose em pimentões, de forma que se possa tomar medidas efetivas para o controle e combate do fungo.

Este trabalho está divido em seis seções: Seção 2 descreve alguns trabalhos relacionados, Seção 3 abordará um pouco sobre a doença antracnose do pimentão. A Seção 4 apresenta um referencial teórico com os principais conceitos sobre a lógica $F u z z y$, na Seção 5 será descrita a abordagem proposta no trabalho, na Seção 6 os resultados e discussões e por fim, na Seção 7 é descrita a conclusão e os trabalhos futuros.

\section{Trabalhos Relacionados}

Esta seção apresenta uma revisão bibliográfica de forma reflexiva sobre diferentes abordagens dentro do escopo da Inteligência Artificial descrevendo suas principais características.

[de Nazaré Júnior et al. 2009] propôs um método para a detecção e quantificação automática da área foliar danificada por pragas usando imagens digitais. A partir da reconstrução das estruturas danificadas das folhas e de áreas danificadas estimadas. $\mathrm{O}$ método proposto melhorou em $40 \%$ o erro absoluto gerado por um método da literatura a partir da utilização de técnicas de Inteligência Artificial.

[GALVÃO et al. 2018] propos auxiliar os agricultores no combate contra duas doenças cancerígenas frequentes em plantações de café, a cercosporiose e a ferrugem. Com o auxílio de técnicas de visão computacional, aprendizado de máquina através de redes neurais e reconhecimento de padrões desenvolveu um algoritmo capaz de identificar plantas que contenham as doenças mencionadas.

[de Sousa et al. 2019] realizou um estudo por meio da implementação da rede neural RBF, objetivando utilizá-la como ferramenta de apoio no diagnóstico doença $\mathrm{Hu}$ anglongbing em um pomar de tangerina poncã. Utilizou uma base de dados populada com 29 amostras e 8 atributos, com ênfase no treinamento e teste para classificação das amostras utilizadas. Os resultados foram analisados estatisticamente obtendo-se uma acurácia média de $77,4 \%$;

[Ferreira et al. 2019] construiu um sistema para auxiliar profissionais na detecção da doença murcha de fusarium no tomateiro através de um sistema fuzzy, antes que a doença se manifeste em todo o plantio. Tinha como objetivo discutir o uso da lógica fuzzy como mecanismo auxiliar na análise do tomateiro para detectar se o mesmo é portador ou pode desenvolver a doença futuramente. 


\section{Antracnose}

A antracnose nos pimentões é vista como uma das doenças mais destrutivas com perdas significativas na produção [Reis et al. 2009]. É uma das mais importantes doenças dessa hortaliça em regiões de temperaturas entre 20 e $25{ }^{\circ} \mathrm{C}$, e umidade relativa do ar elevada [Viana et al. 2007]. No Brasil perdas de até $100 \%$ têm sido relatadas em cultivares de pimentão e pimentas [Reis et al. 2009].

\subsection{Sintomas}

Solanáceas como o pimentão apresentam sintomas de antracnose em toda a parte aérea, porém nos frutos os sintomas são mais expressivos [Bueno et al. 2006]. Na folhas e nos ramos, os sintomas são evidenciados pela presença de pequenas lesões necróticas de contornos circulares alongados [Tozze Junior 2007].

É comum observar grande quantidade de frutos com infecção latente nos quais os sintomas se manifestam apenas alguns dias após a colheita [Reis et al. 2009]. Nos frutos, onde as lesões são mais típicas, essas são deprimidas, circulares, de bordos elevados e de diferentes tamanhos. Nessas lesões, sob condições de elevada umidade, o patógeno produz frutificações na forma de acérvulos escuros com massas de conídios de coloração rósea, salmão ou alaranjada, as quais se destacam no verde dos frutos [Viana et al. 2007]. Assim a Figura 1 mostra frutos acometidos pela doença demonstrando os sintomas acima relatados.

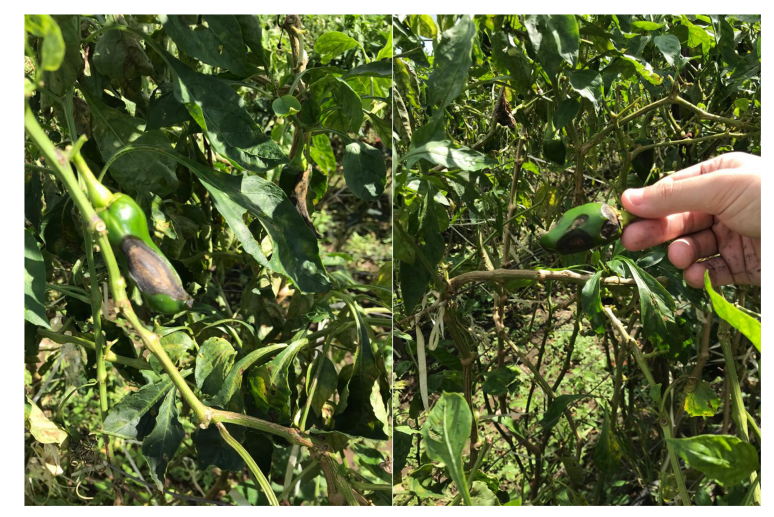

Figura 1. Frutos Acometidos Pela Antracnose

\subsection{Meios de Transmissão}

Os conídios (estruturas de multiplicação dos fungos) são disseminados pelos respingos da água da chuva e da irrigação por aspersão. A longa distância, a disseminação se dá por meio das sementes [GASPAROTTO et al. 2014], sobrevivendo ainda em restos de culturas e em outras hospedeiras da família, como o jiló, o tomate e a berinjela [Viana et al. 2007].

\section{Lógica Fuzzy}

O termo Fuzzy em língua inglesa pode ter vários significados, que variam de acordo com o contexto de interesse, mas o conceito básico deste adjetivo passa sempre pelo vago, 
indistinto, incerto. As tentativas de tradução para o português ainda não são unanimidades: "nebuloso" e "difuso" são exemplos mais populares de traduções para Fuzzy [Marro et al. 2010].

De acordo com [Gomide and Gudwin 1994] a lógica Fuzzy é a lógica baseada na teoria dos conjuntos Fuzzy. Ela difere dos sistemas lógicos tradicionais em suas características e seus detalhes. Na lógica Fuzzy, o valor verdade de uma proposição pode ser um subconjunto Fuzzy de qualquer conjunto parcialmente ordenado, ao contrário dos sistemas lógicos binários, onde o valor verdade só pode assumir dois valores: verdadeiro ou falso. Nos sistemas lógicos multivalores, o valor verdade de uma proposição pode ser ou um elemento de um conjunto finito, num intervalo, ou uma álgebra booleana [Gomide et al. 1995].

\section{Abordagem Proposta}

Com o auxílio da plataforma MatLab, modelou-se um sistema com a utilização da lógica Fuzzy para a realização de pré-diagnósticos, onde foi utilizado como dados de entrada características associadas a antracnose, salientando-se que uma base de dados foi coletada pelos autores. Todos os sintomas utilizados apresentam as seguintes fases: leve, moderada e grave.

\subsection{Base de dados}

Os dados foram coletados na cidade de Tianguá-CE, na zona rural conhecido como Sítio Córrego. As amostras de dados foram montadas a partir de 71 plantas e 368 amostras de frutos, destas amostras foram analisados 9 características que foram divididas em 3 grupos. Cada grupo de características separadas por partes da planta: caule, folhas e frutos. Começando pelo caule, as propriedades observadas foram: lesões escuras nas hastes, necrose e lesões em forma de estrias. Enquanto que nas folhas foram observados: manchas circulares, manchas necróticas, folhas irregulares com coloração parda. Por fim no fruto: lesões nos frutos, necrose nos botões florais, lesão circular de bordos elevados.

\subsection{Sistema Fuzzy}

Modelou-se o sistema com a utilização da Lógica Fuzzy através do software MatLab, para a realização dos diagnósticos usando amostras da base de dados coletada, os sintomas que estão na base de dados podem apresentar as fases: leve, media e grave. A Figura 2 mostra a declaração das variáveis na ferramenta MatLab, nela são utilizadas nove variáveis de entrada correspondentes aos sintomas necessários para a identificação da doença antracnose.

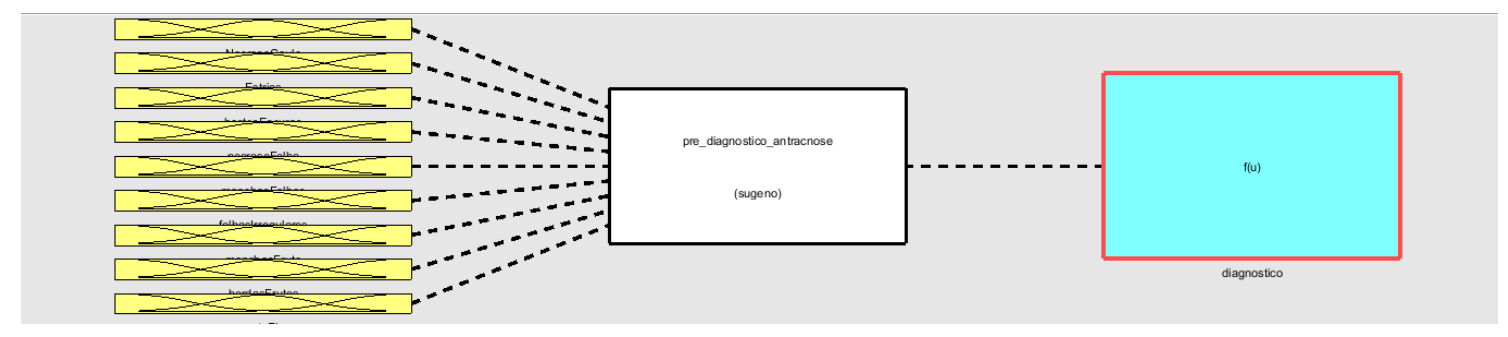

Figura 2. Variáveis de Entrada 
A Figura 3 demonstra a definição das regras as quais são necessárias para obter o diagnóstico da antracnose. Foram elaboradas 689 regras de inferência para obter todos os possíveis resultados de combinações entre as entradas e as saídas esperadas. A partir destas regras é possível identificar se a planta analisada tem ou não o risco de ter a doença e o nível de gravidade.

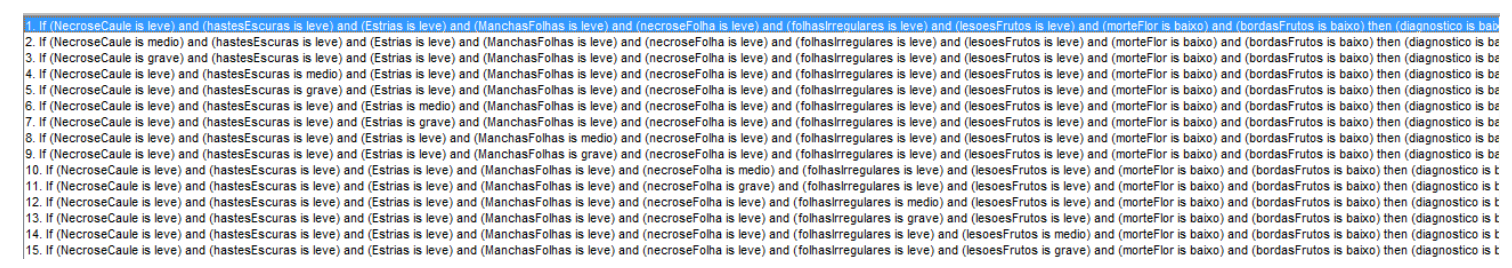

Figura 3. Regras de Inferência do Sistema Fuzzy

\section{Resultados e Discussões}

A Tabela 1 mostra os sintomas e as gravidades de uma das amostras coletadas, a qual foi verificado que alguns apresentaram os sintomas na fase leve, tais como: necrose no caule apresentando 0.29, lesões escuras nas hastes com 0.2, manchas circulares nas folhas 0.17 . Porém outros sintomas apresentaram a fase mediana com aproximação para grave assim como: manchas necróticas nas folhas com 0.64 , lesões nos frutos 0.63 . Por fim foram observados os seguintes sintomas em sua fase grave: lesões em forma de estrias 0.84 , folhas irregulares e coloração parda com 0.77 , necrose nos botões florais com 0.74 , lesão circular de bordos elevados com 0.77 .

Tabela 1. Primeiro Diagnóstico

\begin{tabular}{|l|c|}
\hline Sintomas & Gravidade \\
\hline Necrose no caule & 0.29 \\
\hline Lesões escuras nas hastes & 0.31 \\
\hline Lesões em forma de estria & 0.84 \\
\hline Manchas circulares nas folhas & 0.17 \\
\hline Manchas necróticas nas folhas & 0.64 \\
\hline Folhas irregulares e pardas & 0.77 \\
\hline Lesões nos frutos & 0.63 \\
\hline Necrose dos botões florais & 0.74 \\
\hline Lesão circular nos frutos & 0.77 \\
\hline
\end{tabular}

Na Figura 4 são apresentados os resultados obtidos com a realização de um diagnóstico da antracnose, obtido através dos resultados de todos os sintomas aplicando as regras de inferência do sistema Fuzzy. Assim o resultado obtido foi de 0.75 demonstrando que a planta se encontra com alto risco de ter a doença.

A Tabela 2 mostra os sintomas e a gravidade de outra amostra coletada, a qual foi verificado que alguns apresentaram-se na fase leve, tais como: necrose no caule apresentando 0.32 , lesões escuras nas hastes com 0.26 , folhas irregulares e coloração parda com 0.3 , lesão circular de bordos elevados com 0.28 . Porém outros sintomas apresentaram-se 


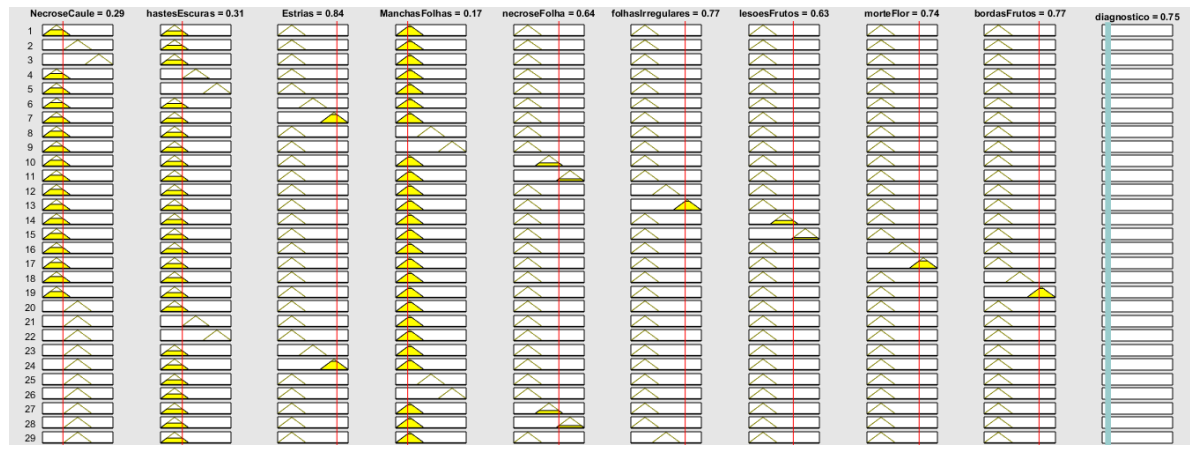

Figura 4. Primeiro Diagnóstico

na fase mediana como: manchas circulares nas folhas 0.37 , manchas necróticas nas folhas com 0.47 , necrose nos botões florais com 0.32 , lesões nos frutos 0.36 . Por fim foram observados os seguintes sintomas na fase grave: lesões em forma de estrias 0.75 .

Tabela 2. Segundo Diagnóstico

\begin{tabular}{|l|c|}
\hline Sintomas & Gravidade \\
\hline Necrose no caule & 0.32 \\
\hline Lesões escuras nas hastes & 0.26 \\
\hline Lesões em forma de estrias & 0.75 \\
\hline Manchas circulares nas folhas & 0.37 \\
\hline Manchas necróticas nas folhas & 0.47 \\
\hline Folhas irregulares e pardas & 0.3 \\
\hline Lesões nos frutos & 0.36 \\
\hline Necrose dos botões florais & 0.32 \\
\hline Lesão circular nos frutos & 0.28 \\
\hline
\end{tabular}

Na Figura 5 são apresentados os resultados obtidos com base no diagnóstico utilizando como apoio todos os sintomas baseado nas regras de inferência do sistema Fuzzy, obtendo-se 0.17 , evidenciando que a planta encontra-se com risco muito baixo de ter a doença.

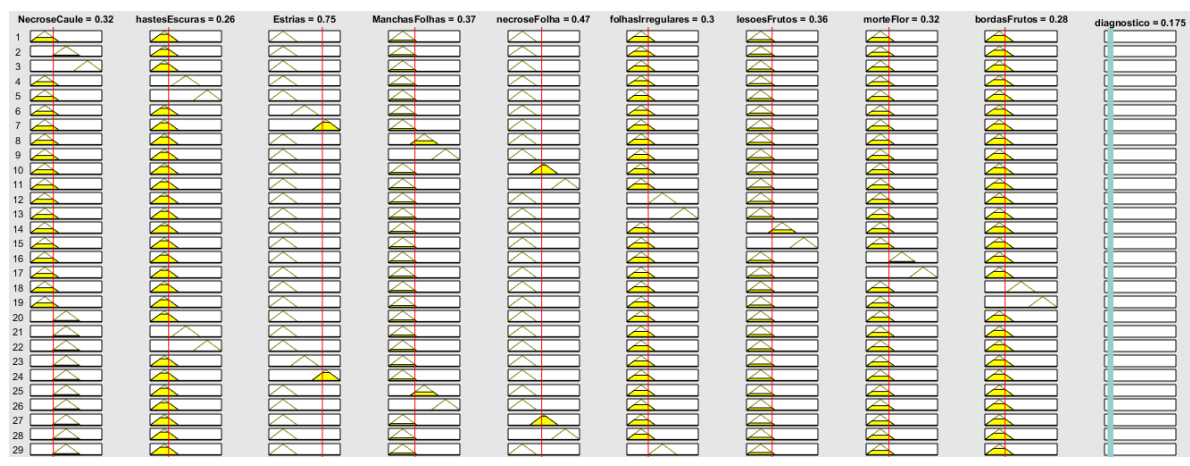

Figura 5. Segundo Diagnóstico

Já a Tabela 3 evidencia os sintomas e a gravidade de cada uma das amostras coletadas, na fase leve, tais como: manchas circulares nas folhas 0.25 , necrose nos botões 
florais com 0.29. Outros sintomas apresentaram-se na fase mediana como: necrose no caule apresentando 0.45 , lesões escuras nas hastes com 0.58 , lesões em forma de estrias 0.54, manchas necróticas nas folhas com 0.58 , lesões nos frutos 0.42 , lesão circular de bordos elevados com 0.67 . Por fim sintomas na fase grave: folhas irregulares e coloração parda com 0.8

Tabela 3. Terceiro Diagnóstico

\begin{tabular}{|l|c|}
\hline Sintomas & Gravidade \\
\hline Necrose no caule & 0.45 \\
\hline Lesões escuras nas hastes & 0.58 \\
\hline Lesões em forma de estrias & 0.54 \\
\hline Manchas circulares nas folhas & 0.25 \\
\hline Manchas necróticas nas folhas & 0.58 \\
\hline Folhas irregulares e pardas & 0.8 \\
\hline Lesões nos frutos & 0.42 \\
\hline Necrose dos botões florais & 0.29 \\
\hline Lesão circular nos frutos & 0.67 \\
\hline
\end{tabular}

Na Figura 6 observa-se os resultados obtidos com a realização do diagnóstico obtido com base nos resultados de todos os sintomas aplicando as regras de inferência do sistema Fuzzy. Assim obteve-se 0.5, concluindo então que a planta encontra-se com risco mediano de ter a doença.

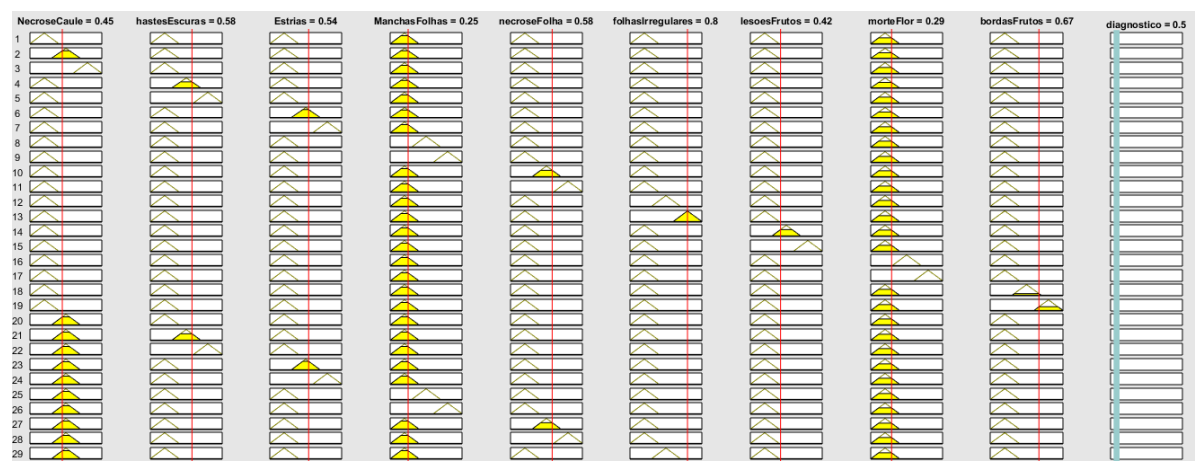

Figura 6. Terceiro Diagnóstico

\section{Conclusão e Trabalhos Futuros}

A Antracnose é uma doença que causa danos a economia prejudicando os produtores de hortaliças. Este trabalho evidencia que a lógica Fuzzy é um método que pode ser utilizado como um recurso auxiliador na predição doenças. Podendo ajudar profissionais da agricultura a diagnosticarem a presença da doença em cultivares como o pimentão, contribuindo para que a planta possa ter o tratamento mais indicado, garantindo o máximo de eficiência na produção de alimentos.

Como trabalho futuro sugere-se a aplicação da lógica Fuzzy como recurso auxiliar no diagnóstico da requeima em tomates, doença que provoca várias perdas na produção gerando assim grandes prejuízos econômicos aos produtores. 


\section{Referências}

Bueno, C. R. N. C., Junior, M., and Sidnei, N. (2006). Identificação e caracterização das espécies de colletotrichum causadoras de antracnose em hortaliças solanáceas.

da Silva Albuquerque, F., de França, Ê. F., de Souza, A. E. R., Rolim, M. M., et al. (2011). Lixiviação de potássio em um cultivo de pimentão sob lâminas de irrigação e doses de potássio. Revista Caatinga, 24(3):135-144.

de Azevedo, C. P., Café Filho, A., and REIS, A. (2006). Recomendações de manejo da antracnose do pimentão e das pimentas. Embrapa Hortaliças-Comunicado Técnico (INFOTECA-E).

de Nazaré Júnior, A. C., Menotti, D., Neves, J. M. R., and Sediyama, T. (2009). Detecção automática da área foliar danificada da soja através de imagens digitais. XVIII SEIC/UFOP, page 8 .

de Sousa, R. N., de Brito, R. X., and de Sousa Ximenes, J. N. (2019). Aplicação da rede neural rbf como mecanismo auxiliador no diagnóstico da doença hlb em espécies citricas. In Anais da VII Escola Regional de Computação Aplicada à Saúde, pages 55-60. SBC.

Ferreira, A. F. G., Moreira, L. F., de Arruda, G. R., de Brito, R. X., and Ferreira, A. V. G. (2019). Sistema fuzzy como ferramenta auxiliar na detecção da murcha de fusarium no tomateiro. In Anais da VII Escola Regional de Computação Aplicada à Saúde, pages 193-198. SBC.

GALVÃO, G. F. P., Carvalho, W., Rocha, W., and da Silva Costa, J. C. (2018). Visão computacional para detecção de doenças fúngicas na agricultura. ÚNICA Cadernos Acadêmicos, 2(1).

GASPAROTTO, L., Pereira, J., and Berni, R. (2014). A antracnose da pimenta-de-cheiro. Embrapa Amazônia Ocidental-Comunicado Técnico (INFOTECA-E).

Gomide, F., Gudwin, R. R., and Tanscheit, R. (1995). Conceitos fundamentais da teoria de conjuntos fuzzy, lógica fuzzy e aplicações. In Proc. 6 th IFSA Congress-Tutorials, volume 59, pages $1-38$.

Gomide, F. A. C. and Gudwin, R. R. (1994). Modelagem, controle, sistemas e lógica fuzzy. SBA controle \& Automação, 4(3):97-115.

Marro, A. A., Souza, A. d. C., Cavalcante, E. d. S., Bezerra, G. S., and NUNES, R. O. (2010). Lógica fuzzy: conceitos e aplicações. Natal: Universidade Federal do Rio Grande do Norte (UFRN).

Reis, A., Boiteux, L. S., and Henz, G. P. (2009). Antracnose em hortaliças da família solanacea. Embrapa Hortaliças-Circular Técnica (INFOTECA-E).

Tozze Junior, H. J. (2007). Caracterização e identificação de espécies de Colletotrichum associadas à antracnose do pimentão (Capsicum annuum) no Brasil. $\mathrm{PhD}$ thesis, Universidade de São Paulo.

Viana, F., FREIRE, F., and Parente, G. (2007). Controle das principais doenças do pimentão cultivado nas regiões serranas do estado do ceará. Embrapa Agroindústria Tropical-Comunicado Técnico (INFOTECA-E). 